\title{
Clinical Manifestations, Fluid Changes and Neuroimaging Alterations in Patients with General Paresis of the Insane
}

This article was published in the following Dove Press journal: Neuropsychiatric Disease and Treatment

\author{
Jun-Hua Gao' \\ Wu-Rong $\mathrm{Li}^{\prime}$ \\ Dong-Mei $X u^{\prime}$ \\ Bo-Wen Zheng' \\ Yu-Ming Huang' \\ Wen-Qing $\mathrm{Wu}^{\prime}$ \\ Wei Zhang ${ }^{2}$
}

'Department of Neurology, Beijing Ditan Hospital, Capital Medical University, Beijing, People's Republic of China; ${ }^{2}$ Center for Cognitive Neurology, Department of Neurology, Beijing Tiantan Hospital, Capital Medical University, Beijing, People's Republic of China
Correspondence: Wei Zhang

Center for Cognitive Neurology,

Department of Neurology, Beijing Tiantan

Hospital, Capital Medical University, Beijing

10070, People's Republic of China

Email ttyyzw@163.com
Purpose: We aim to study the clinical manifestations, fluid changes and neuroimaging alterations in patients with general paresis of the insane (GPI).

Methods: A total of 119 patients suffering from GPI recruited in Beijing Ditan Hospital, Capital Medical University from 2010 to 2020 were retrospectively analyzed.

Results: In 119 GPI patients, 103 cases $(86.6 \%)$ were male. Misdiagnosed rate was up to $83.2 \%$, schizophrenia and mood disorders were the most common misdiagnosed diseases. Duration from symptom onset to the final confirmed diagnosis was $10.4 \pm 12.9$ months. The main clinical manifestations included cognitive impairment (114 cases, 95.8\%) and neuropsychiatric symptoms (107 cases, 90.0\%). The cognitive domains including the delayed recall, visuospatial/executive function and language ability indicated by MoCA score were damaged severely. Rapid plasma regain (RPR) of all GPI patients was $100 \%$ positive in serum and $89.9 \%$ positive in cerebral spinal fluid (CSF). The white blood cell (WBC) number in CSF was between 6 and $50 / \mu \mathrm{L}$ in 73 GPI patients (61.3\%). The protein level was between 45.1 and $70 \mathrm{mg} / \mathrm{dL}$ in 47 cases $(39.5 \%)$. In the 110 cases, 96 cases $(87.3 \%)$ were abnormal indicated by cerebral atrophy mostly located in the anterior brain and abnormal signals distributed in various regions of the brain mostly in the frontal lobe and temporal lobe.

Conclusion: The symptoms of GPI were complex and easy to misdiagnose. The clinicians were still short of vigilance for neurosyphilis. We should expand serologic testing for syphilis especially in patients with cognitive impairment and neuropsychiatric symptoms. We suggest syphilis curricula in the training program of the clinicians especially for neurologist and psychiatrist.

Keywords: dementia, clinical manifestation, fluid changes, neuroimaging alterations, general paresis of the insane

\section{Introduction}

Syphilis is caused by Treponema pallidum, subspecies pallidum (T. pallidum) and the incidence is rising year by year in China. It was reported by the Chinese Center for Disease Control and Prevention that there were reported 494,867 cases of syphilis nationwide with the incidence of $35.62 / 100,000$ in 2018 , which was significantly higher than that of $6.43 / 100,000$ in 2000. Furthermore, T. pallidum can invade the brain and cause neurosyphilis, with an incidence of $0.47-2.1$ cases/ $100,000 .^{1,2}$ Neurosyphilis is divided into early and late forms and presents many symptoms and signs during the whole process of the disease. General paresis of the insane (GPI), also termed dementia paralytica, is one of the most common late 
forms of neurosyphilis, accounting for about $38.9-49.0 \%$ of total neurosyphilis reported by other investigations $\mathrm{s}^{3,4}$ and $40.0 \%$ by our previous study. ${ }^{5}$

Until now, no larger sample study on the features of GPI patients was reported abroad. There were only two studies from China focusing on the changes of clinical and auxiliary examination of GPI patients with $116^{6}$ and 85 patients, ${ }^{7}$ respectively. Data from the two studies showed the various clinical manifestations of GPI. In addition, although cognitive impairment and neuropsychiatric symptoms were considered as the common manifestations of GPI, objective assessment such as specific rating scales were not used. Furthermore, the two studies failed to investigate the number of white blood cells (WBC) and level of protein in cerebrospinal fluid (CSF). Moreover, although the two studies assessed the brain changes by brain magnetic resonance imaging (MRI) or computerized tomography (CT) and all found brain atrophy in $87.1 \%$ and $56.1 \%$ of GPI patients, respectively, they did not analyze the brain regions that were prone to be affected.

The purpose of this study was to determine the clinical manifestations of GPI patients and analyze the brain changes by MRI, thus providing evidence from multiple aspects for early diagnosis and timely treatment.

\section{Methods}

We retrospectively collected the GPI patients through the electronic medical record (EMR) system in Beijing Ditan Hospital from January 2010 to January 2020. Because of the retrospective nature of the study, patient consent for inclusion was waived. We managed the files strictly and hid the relevant information of the patient, including name, home address, and telephone number, etc. All the authors from Beijing Ditan Hospital collected the clinical data. Occasionally incomplete data emerged. In order to improve data quality and integrity, we would offer the admission date and discharge date of required patient which was routinely recorded on the first page of the medical record to the attending physician. The attending physician who took care of the specific patient during hospitalization would take responsibility to connect patients or their caregivers according to original registration, conduct follow-ups and inquiries, complete required data and obtain details as much as possible. The study was conducted in accordance with the Declaration of Helsinki. It was approved by the Ethical Review Board of Beijing Ditan Hospital, Capital Medical University, the ethics number was Jingdi Lunke Zi (2020) No. (044)-01.
RPR in CSF is recommended as the diagnosis standard by European guideline. ${ }^{8}$ GPI was diagnosed according to the European Centers for Disease Control (CDC) ${ }^{8}$ guideline and US guideline ${ }^{9}$ and as follows: 1 . Syphilis at any stage meets: (1) a positive RPR in CSF, or (2) positive T. pallidum particle agglutination (TPPA) and fluorescent treponemal antibody absorption (FTA-ABS) in CSF, with increased WBC number $(>5 / \mu \mathrm{L})$ or protein level $(>45 \mathrm{mg} / \mathrm{dL})$ in CSF, and with no other known causes inducing these abnormalities. 2. Dementia met the Diagnostic and Statistical Manual of Mental Disorder, 4th ed (DSM-IV). The exclusion criteria of GPI included: 1. HIV-positive patients. 2. The presence of neurological disorders besides neurosyphilis that might affect cognition, such as Alzheimer's disease, vascular diseases, folic acid deficiency, alcoholism, etc. 3. The presence of psychiatric disorders, such as schizophrenia and depression, etc.

\section{Collection of Clinical Data}

Clinical data of all GPI patients, including demographic information, symptoms and signs, past medical history, the first diagnosis given by doctor, the results of GPI-related variables and neuroimage presentations by MRI were systemically reviewed.

\section{Evaluation of Cognitive Function and Neuropsychiatric Symptoms}

Mini-mental State Examination (MMSE) and Montreal Cognitive Assessment (MoCA) scales were used to evaluate the overall cognitive function. MMSE is an internationally recognized scale for assessing overall cognitive function. ${ }^{10}$ The cutoff point of MMSE was based on the education level, just as illiteracy $\leq 17$ points, primary school education $\leq 20$ points and secondary school education $\leq 24$ points were considered as abnormal. ${ }^{11}$

MoCA is another scale rating the global cognitive function. The cutoff point of MoCA was 26 points when the educational level is $\geq 12$ years. It contains multiple cognitive domains, including orientation, memory, calculation, language and visuospatial ability. ${ }^{12}$ In this study, the score of each cognitive domain in MoCA was presented. The full mark rate was calculated as the numbers of GPI patients with full score of each domain divided by the total cases.

We utilized the Neuropsychiatric Inventory (NPI) scale for assessing the neuropsychiatric symptoms for dementia patients, which contains 12 items, including delusion, 
hallucination, agitation, dysphoria, anxiety, apathy, irritability, euphoria, disinhibition, aberrant motor activity, abnormal sleeping, and eating symptoms. According to the NPI reports, neuropsychiatric symptoms were identified in our study.

\section{Collections of CSF and Serum and Measurements of GPI-related Variables}

RPR and TPPA results in both serum and CSF of all the GPI patients were collected. WBC number and protein level in CSF were summarized too.

\section{Neuroimaging Examination}

In this study, 110 GPI patients finished brain 1.5 or $3.0 \mathrm{~T}$ MRI scan (Siemens Company, Germany), including T1weighted spin echo, T2-weighted spin echo, and fluid attenuated inversion recovery (FLAIR). The parameters of MRI were as follows: repetition time (TR): $2300 \mathrm{~ms}$, echo time (TE): $2.3 \mathrm{~ms}$, layer thickness: $1 \mathrm{~mm}$. The MRI results were independently interpreted by two neuroimaging experts from Beijing Ditan hospital.

\section{Results}

\section{Demographic Information}

In this study, a total of 119 GPI patients were recruited. The gender ratio of male to female was 6.4:1. 113 cases were married. The educational level of most of GPI patients $(n=104,87.4 \%)$ was higher than elementary school. Two college students were diagnosed with GPI. They all denied sexual life and their parents were all with TPPA positive in serum. It took 20 months and 40 months for the two students to make the final diagnosis after the first time of visiting doctor. The average time from symptoms onset to the final confirmed diagnosis was $10.4 \pm 12.9$ months. A total of nine patients had a history of syphilis when they first saw the doctor in the other hospitals. (Table 1).

\section{Clinical Characteristics}

It was found that cognitive impairment $(n=114,95.8 \%)$ and neuropsychiatric symptoms $(n=107,89.9 \%)$ were the main manifestations of GPI patients.

\section{Cognitive Assessment}

In 119 GPI patients, there were 74 cases fulfilled MMSE, while 41 cases fulfilled MoCA. According to the full mark rate from low to high, the damaged cognitive domains in
Table I The Demographic Information of GPI Patients

\begin{tabular}{|l|l|l|}
\hline & $\begin{array}{l}\text { GPI Cases } \\
\text { (n=I I9) }\end{array}$ & $\begin{array}{l}\text { \% of Total } \\
\text { Cases }\end{array}$ \\
\hline $\begin{array}{l}\text { Age (years, mean } \pm \text { SD) } \\
\text { Male }\end{array}$ & $\begin{array}{l}51.8 \pm 10.7 \\
103\end{array}$ & 86.6 \\
\hline Marital status & & \\
$\quad$ Married & 113 & 95.0 \\
Unmarried & 6 & 5.0 \\
\hline Education level & & \\
Elementary school & 15 & 12.6 \\
Junior high school & 60 & 50.4 \\
Senior high school & 23 & 19.3 \\
Junior college & 21 & 17.7 \\
\hline Occupations & & \\
Worker & 44 & 37.0 \\
Peasant & 15 & 12.6 \\
Manager & 15 & 12.6 \\
Driver & 10 & 8.4 \\
Civil servant & 9 & 7.6 \\
Self-employed & 8 & 6.7 \\
Student & 2 & 1.7 \\
Others & 17 & 14.3 \\
\hline History of syphilis & 96.6 \\
Treated with penicillin before & $10.4 \pm 12.9$ & \\
Duration from onset to diagnosis & & \\
(month, mean $\pm S D)$ & & \\
\hline
\end{tabular}

MoCA were delayed recall, visuospatial/executive and language ability in GPI patients (Table 2).

\section{Neuropsychiatric Symptoms}

According to the occurrence rate of 12 items in NPI from high to low, the neuropsychiatric symptoms were irritability, agitation/aggression, aberrant motor behavior and abnormal sleeping. Apathy and abnormal eating symptom were rare in GPI patients (Table 3).

\section{Other Neurological Manifestations}

There were a variety of other symptoms and signs in GPI patients (Table 4). As for the symptoms, dysarthria was the most common manifestation with frequency of $20.2 \%$, followed by epilepsy $(9.2 \%)$ and urinary incontinence $(9.2 \%)$. As for the signs, pupillary abnormality was the common sign in this study, including Argyll Robertson pupil (23.5\%) and other abnormal pupils, such as pupil shape and size change $(13.5 \%)$. Optic atrophy was one type of parenchymal neurosyphilis, which could be an accompanying sign of GPI with a frequency of $3.4 \%$. In dyskinesia, candy sign $(22.7 \%)$ was 
Table 2 Evaluation of Cognitive Function for GPI Patients

\begin{tabular}{|l|l|l|l|}
\hline & Number of Cases (n) & Score (mean \pm SD) & Abnormal Rate (\%) \\
\hline MMSE & 74 & $21.3 \pm 5.7$ & 54.1 \\
MoCA & $4 \mathrm{I}$ & $16.1 \pm 5.8$ & 95.1 \\
\hline Cognitive domains & & & Full mark rate (\%) \\
Delayed recall & $4 \mathrm{I}$ & $0.9 \pm 1.3$ & 2.4 \\
Visuospatial/executive & $4 \mathrm{I}$ & $2.2 \pm 1.4$ & 4.9 \\
Language capacity & $4 \mathrm{I}$ & $0.9 \pm 0.9$ & 7.3 \\
Abstraction ability & $4 \mathrm{I}$ & $0.6 \pm 0.7$ & 14.6 \\
Orientation & $4 \mathrm{I}$ & $4.0 \pm 1.9$ & 24.3 \\
Attention & $4 \mathrm{I}$ & $4.3 \pm 1.5$ & 26.8 \\
Naming capacity & $4 \mathrm{I}$ & $2.2 \pm 1.0$ & 48.8 \\
\hline
\end{tabular}

Table 3 Elevation of Neuropsychiatric Symptoms for GPI Patients

\begin{tabular}{|l|l|l|}
\hline I 2 Items of NPI & $\begin{array}{l}\text { Number of Cases } \\
\text { (n) }\end{array}$ & $\begin{array}{l}\text { \% of Total } \\
\text { Cases }\end{array}$ \\
\hline Irritability & 75 & 63.0 \\
Agitation/aggression & 50 & 42.0 \\
Aberrant motor behavior & 45 & 37.8 \\
Abnormal sleeping & 45 & 37.8 \\
Hallucination & 44 & 37.0 \\
Depression & 33 & 27.7 \\
Delusion & 32 & 26.9 \\
Disinhibition & 24 & 20.2 \\
Euphoria & 20 & 18.7 \\
Anxiety & 13 & 12.1 \\
Apathy & 10 & 9.3 \\
Abnormal eating symptom & 8 & 7.5 \\
\hline
\end{tabular}

the most common sign, followed by gait instability $(20.2 \%)$, hypermyotonia (11.8\%) and bradykinesia (9.2\%). The frequency of hyperreflexia in this study was $10.9 \%$.

\section{The Misdiagnosed Diseases of GPI}

\section{Patients}

In 119 GPI patients, 99 cases (83.2\%) were wrongly diagnosed as other diseases before the confirmed diagnosis. The misdiagnosed diseases that ranked the top five were schizophrenia, mood disturbances (anxiety and depression), cerebral infarction, vascular dementia, and menopausal symptom (Table 5).

\section{Changes of Syphilis-related Variables in Serum and CSF}

In serum, the results of syphilis-related variables revealed that TPPA and RPR were all positive in 119 GPI patients. In
CSF, TPPA was positive in 119 GPI patients and RPR was positive in 107 cases (89.9\%). Among the 12 cases with negative RPR, eight cases had been treated with benzathine penicillin and RPR titer might be lowered or even disappeared. The RPR titers in both serum and CSF are displayed in Figure 1. There were 43 cases (36.1\%) with serum RPR titer $\leq 1: 16$. However, there were only 58 cases $(48.7 \%)$ with the CSF RPR titer $\geq 1: 4$ (Figure 1).

We summarized the WBC number and protein level in CSF samples of all the 119 GPI patients. The data indicated that 24 cases $(20.2 \%)$ had both normal WBC and protein, 68 cases $(57.1 \%)$ had both increased $\mathrm{WBC}$ and elevated protein, 91 cases $(76.5 \%)$ had increased WBC, and 93 cases $(78.2 \%)$ had elevated protein (Figure 2).

There were 73 cases $(61.3 \%)$ with increased WBC between 6 and $50 / \mu \mathrm{L}$ of the 91 cases, and 15 cases $(12.6 \%)$ with WBC between 50 and $100 / \mu \mathrm{L}$, only 3 cases with WBC more than $100 / \mu \mathrm{L}$, there were 47 cases $(39.5 \%)$ with the elevated protein between 45 and $70 \mathrm{mg} / \mathrm{dL}, 25$ cases $(21.0 \%)$ with protein between 70.1 and $100 \mathrm{mg} / \mathrm{dL}$. The other 21 cases (17.6\%) with protein more than $100 \mathrm{mg} / \mathrm{dL}$.

\section{Electroencephalogram (EEG) Examination} In 119 GPI patients, 84 cases $(70.6 \%)$ finished EEG, in which, 49 cases $(58.3 \%)$ showed normal EEG activity and 19 cases $(22.6 \%)$ showed slightly abnormal EEG activity with mild increased $\theta$ or $\delta$ waves, 16 cases $(19.0 \%)$ showed moderate to severe EEG with diffused $\theta$ or $\delta$ waves. Of the 11 cases with epilepsy, 5 cases showed abnormal waves such as spike or wave discharges. 
Table 4 Frequencies of Other Symptoms and Signs of GPI Patients

\begin{tabular}{|c|c|c|}
\hline & Number of Cases & $\%$ of Total Cases \\
\hline \multicolumn{3}{|l|}{ Symptoms } \\
\hline Dysarthria & 24 & 20.2 \\
\hline Epilepsy & 11 & 9.2 \\
\hline Urinary incontinence & 11 & 9.2 \\
\hline \multicolumn{3}{|l|}{ Signs } \\
\hline \multicolumn{3}{|l|}{ Pupil } \\
\hline Argyll Robertson pupil & 28 & 23.5 \\
\hline Other abnormal pupils & 16 & 13.5 \\
\hline Optic atrophy & 4 & 3.4 \\
\hline \multicolumn{3}{|l|}{ Dyskinesia } \\
\hline Candy sign & 27 & 22.7 \\
\hline Gait instability & 24 & 20.2 \\
\hline Hypermyotonia & 14 & 11.8 \\
\hline Bradykinesia & 11 & 9.2 \\
\hline Hyperreflexia & 13 & 10.9 \\
\hline
\end{tabular}

Table 5 The Misdiagnosed Diseases of 99 GPI Patients

\begin{tabular}{|l|l|l|}
\hline & $\begin{array}{l}\text { Number of } \\
\text { Cases }\end{array}$ & $\begin{array}{l}\text { \% of Total } \\
\text { Cases }\end{array}$ \\
\hline Schizophrenia & 30 & 30.3 \\
Mood disturbances (anxiety and & 30 & 30.3 \\
depression) & & \\
Cerebral infarction & 12 & 12.1 \\
Vascular dementia & 8 & 8.1 \\
Menopausal symptom & 4 & 4.0 \\
Alzheimer's disease & 3 & 3.0 \\
Hydrocephalus & 3 & 3.0 \\
Viral encephalitis & 3 & 3.0 \\
Alcoholic encephalopathy & 2 & 2.0 \\
Leukoencephalopathy & 2 & 2.0 \\
Epilepsy & $\mathrm{I}$ & 1.0 \\
Frontotemporal dementia & $\mathrm{I}$ & 1.0 \\
\hline
\end{tabular}

\section{Neuroimaging Alterations by MRI}

In 119 GPI patients, 9 patients did not complete brain MRI scan due to various reasons, the other 110 cases finished brain MRI scan, in which 13 cases (11.8\%) were normal and 97 cases $(88.2 \%)$ were abnormal (Table 6). Atrophy was the most common brain changes (60.9\%), temporal lobe ranked the top (59.1\%), followed by frontal lobe (30.0\%) and parietal lobe $(4.55 \%)$. Interestingly, no atrophy was observed in occipital lobe (Table 6). Abnormal signals in MRI were another common brain alteration $(66.4 \%)$, and the brain regions with frequency of abnormal signal from high to low were frontal lobe (25.5\%), temporal lobe (13.6\%), parietal lobe and white matter $(11.8 \%)$, and occipital lobe $(2.7 \%)$. In addition, it was found that 13 cases $(11.8 \%)$ had hydrocephalus in MRI (Table 6).

\section{Discussion}

Parenchymal neurosyphilis usually exists decades after syphilis infection. ${ }^{13}$ GPI is the dominant form of parenchymal neurosyphilis that is easily misdiagnosed because of multiple nonspecific symptoms and signs. It mostly occurred in the middle-aged population, no matter in the pre-antibiotic era ${ }^{14}$ or in the modern era. ${ }^{7}$ It was reported that the gender ratio of male to female in all types of neurosyphilis was about $1.53 \sim 5.1: 1^{4,5}$ and this ratio was even higher in GPI, ${ }^{6,15,16}$ which was the same as our results. In contrast, Tong et al discovered that compared with non-neurosyphilis dementia, GPI had a higher percentage of males and younger age. ${ }^{15}$ We suspected the reason was that syphilis patients increased most among men who had sex with men (MSM) ${ }^{17}$ and the females might have more opportunities (such as pregnancy and abortion) to screen syphilis-related variables detected than the males, thus, they were less likely to develop symptomatic neurosyphilis due to the early diagnosis and treatment. A previous study summarized the GPI patients from 1924-1954 and discovered the low education level of the total patients ( $95 \%$ completed elementary school only). They assumed the reason was that the patients at higher social class had the chance to be treated in private institutes or even at home. ${ }^{14}$ But in this study $87.4 \%$ of patients had a higher education level than elementary school. To our surprise, two college students were included. The improving education level of society could be the reason. The patients with high education level and high social class were all in great danger of suffering from GPI. Besides, sex education is still a critically important public health and policy issue, perhaps short films, educational pamphlets and wax models portraying syphilis would work. ${ }^{18}$ We recommend effective and comprehensive sexuality education in the society.

Unfortunately, delayed diagnosis was found in our study. There were nine GPI cases that had a definite history of syphilis previously. However, four of them were not correctly diagnosed at their first visit to the doctor. And although three cases in the remaining 110 cases were detected positive syphilis when they were admitted in other hospitals, none of them were diagnosed with GPI 


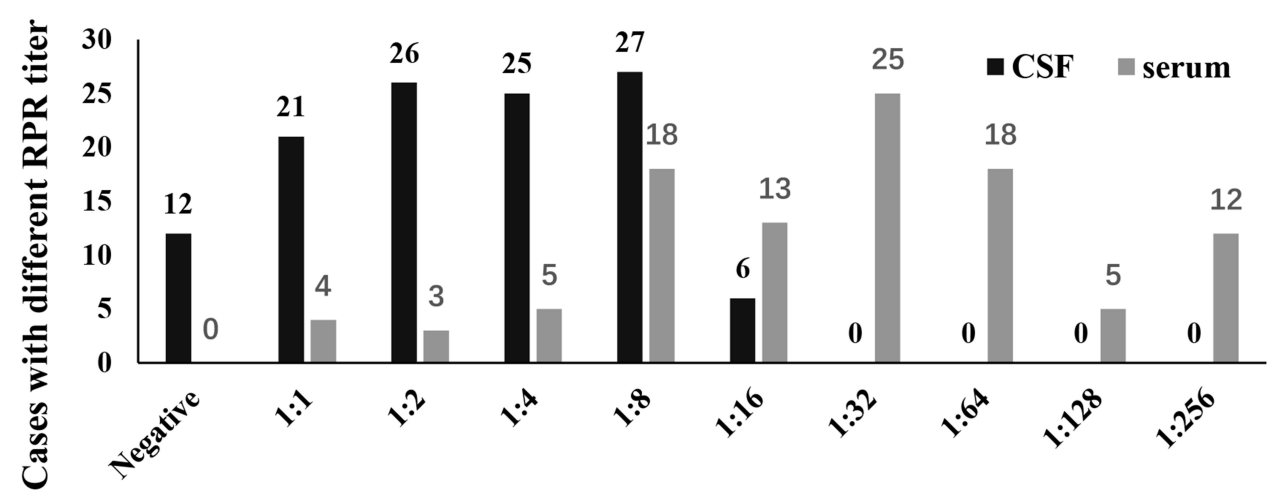

RPR Titer

Figure I RPR titer in serum and CSF from II9 GPI patients. Abbreviation: RPR, rapid plasma regain.

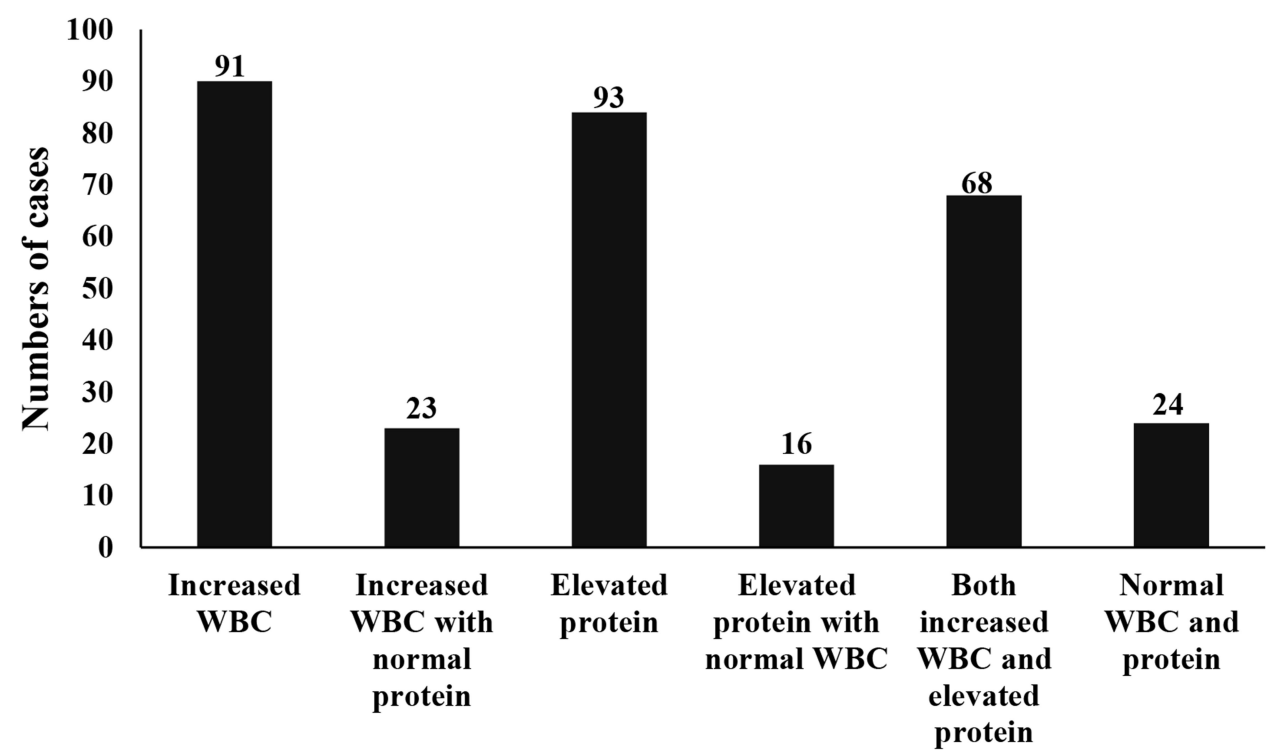

Figure 2 Results of WBC and protein in CSF from II9 GPI patients.

and given related treatment. Previous studies showed the duration from symptom onset to final confirmed diagnosis was about 10-20 months, ${ }^{19-21}$ even after four years of misdiagnosis. ${ }^{22}$ Lack of vigilance for neurosyphilis by the clinicians including the neurologist and psychiatrist prolonged the time from symptom onset to final confirmed diagnosis. We suggest that syphilis curricula should be included in the training program of the clinicians especially neurologist and psychiatrist.

Cognitive impairment was the most common symptom of GPI patients. In this study, most of the GPI patients complained of memory decline, which was easily to be noticed due to its significant impact on their daily life. Furthermore, the abnormal rate of MoCA was much higher than MMSE in our study, This could be because that MoCA was more sensitive for screening out mild cognitive impairment patients and with less ceiling effect. ${ }^{23}$ Further investigation indicated that cognitive domains of delayed recall, visuospatial/executive and language ability were severely damaged. But in the stage of mild GPI, there was an overall cognitive function impairment, just like $\mathrm{AD}$ pattern. ${ }^{16}$ It was known that hippocampus was related to memory and language learning, and left parahippocampal gyrus played an integral role on the visuospatial/executive function. ${ }^{24}$ Thus, we suspected that the temporal lobe might be the earliest damaged brain region in GPI patients.

The incidence of neuropsychiatric symptoms of all types of neurosyphilis varied from $17 \%$ to $51 \%{ }^{25}$ But 
Table 6 Brain MRI Findings of I 10 GPI Patients

\begin{tabular}{|l|l|l|}
\hline & Number of Cases & \% of Total Cases \\
\hline Normal & 13 & 11.8 \\
Brain atrophy & 67 & 60.9 \\
Temporal lobe & 65 & 59.1 \\
Frontal lobe & 33 & 30.0 \\
Parietal lobe & 5 & 4.5 \\
Occipital lobe & 0 & 0.0 \\
Abnormal signal & 73 & 65.5 \\
Frontal lobe & 28 & 25.5 \\
Temporal lobe & 15 & 13.6 \\
Parietal lobe & 13 & 11.8 \\
Occipital lobe & 3 & 2.7 \\
White matter & 13 & 11.8 \\
Hydrocephalus & 13 & 11.8 \\
\hline
\end{tabular}

about $79-82.6 \%$ of total GPI patients had psychiatric symptoms. ${ }^{3,20}$ In this study, the frequency of neuropsychiatric symptoms reached up to $89.9 \%$. Among the 12 neuropsychiatric symptoms in NPI, irritability was the most common symptom, followed by agitation/aggression and aberrant motor behavior. A previous study showed that the incidence of neuropsychiatric symptoms from high to low were agitation/aggression (52.7\%), irritability (47.3\%), apathy (41.8\%) and abnormal sleeping $(38.5 \%) .{ }^{26}$ A report on the misdiagnosed GPI cases discovered that agitation was the most common mood disturbance, ${ }^{20}$ Roberts and Emsley considered agitation as an indicator for the diagnosis of GPI. ${ }^{27}$ The difference of the studies might have GPI patients with different disease severity enrolled. Dopamine pathway ${ }^{28}$ and glutamate signaling $^{29}$ were hypothesized as the main neurotransmitter basis of neuropsychiatric symptoms. ${ }^{25}$ It was confirmed that the frontal lesions had significant associations with the overall degree of psychiatric morbidity. ${ }^{30}$ All the above results suggested that GPI should be considered when patients presented new psychiatric symptom. The basic treatment for neuropsychiatric symptoms was penicillin $\mathrm{G}$, together with other drugs for dealing with the psychiatric manifestations, such as olanzapine and quetiapine. ${ }^{25}$

Except for cognitive impairment and neuropsychiatric symptoms, there were some other neurological symptoms and signs. The sign that was easily overlooked was Argyll Robertson's pupil with the frequency of $23.5 \%$ in GPI patients. It was the typical sign of neurosyphilis, and usually together with tabes dorsalis. ${ }^{31}$ Pupil shape and size change and optic atrophy were common but not specific to GPI. Neurosyphilis might manifest with movement disorders, such as ataxia, parkinsonism, myoclonus, chorea and dystonia, ${ }^{32}$ sometimes presented the symptoms of progressive supranuclear palsy. ${ }^{33}$ Here, it was found that GPI patients had dyskinesia, including gait instability, hypermyotonia, and bradykinesia, which might be due to the damage in the basal ganglia and midbrain. Particularly, the candy sign was a type of dyskinesia described as sucking with lips, jaws and lower facial muscles. It was first reported as a typical sign of neurosyphilis. ${ }^{34}$ In this retrospective study, we might overlook the candy sign in the early years, thus, its actual frequency might be higher than $22.7 \%$. The incidence of hyperreflexia was only $10.9 \%$, which was different from the result from other investigations. ${ }^{32}$ Thus, hyperreflexia might not be a specific sign of GPI. In conclusion, GPI patients can manifest with an array of symptoms and signs, which might be due to the extensive damage caused by syphilis in the brain and spinal cord. In clinical practice, when the clinicians encountered the patients complaining of cognitive impairment and neuropsychiatric symptoms, we should examine the patient carefully, especially pay attention to the specific signs such as the candy sign and Argyll Robertson pupil; this can help us make a direct test for syphilis.

According to the reports, the misdiagnosis rate of GPI is very high at $38.2-100 \%$, ${ }^{7,21,35}$ which was similar to our result that the frequency of misdiagnosis of GPI was $83.2 \%$. It was considered that dementia and schizophrenia were the most frequently misdiagnosed diseases. ${ }^{7}$ In this study, however, the two diseases misdiagnosed with equal frequency were schizophrenia and mood disorders of anxiety and depression, which were followed by cerebral infarction, vascular dementia, etc. Given the high misdiagnosis rate of GPI, we also recommend every patient with neurological or psychiatric symptoms should have routine blood screening tests for syphilis. If the blood TPPA is positive, the lumbar puncture should be performed when it is necessary.

According to the inclusion criteria, all the treponemal tests, including TPPA and FTA-ABS were all positive in both serum and CSF. Serum RPR titer $\geq 1: 32$ can help predict the likelihood of neurosyphilis in HIV negative subjects. ${ }^{36}$ However, about $39 \%$ of total GPI patients were with serum RPR $\leq 1: 16$ in this study. Hence, patients with a low serum RPR titer might not rule out neurosyphilis. CSF RPR titer in most of GPI patients was distributed between 1:1 and 1:8. It was reported that a fourfold decline of RPR titer in CSF but not in serum RPR was a good predictor for therapeutic efficacy in GPI patients with a positive RPR in $\mathrm{CSF}^{37}$ However, when RPR in CSF was lower or even negative, the past medical history, 
symptoms and sign, and CSF routine test were very important in the diagnosis and follow-up of GPI.

CSF pleocytosis and elevated protein can be negative in GPI patients, although WBC number in CSF was increased in most cases in our study. But it was reported that CSF pleocytosis was a sensitive marker for neurosyphilis except tabes dorsalis. ${ }^{38}$ Similarly to tabes dorsalis, GPI was also the late stage of neurosyphilis, thus, the inflammatory response was milder than that in the early stage of the disease. In addition, we found that the protein level in CSF was normal in $22.8 \%$ of total GPI patients, which was similar to other studies within $25-38.8 \%$ normal protein level. ${ }^{7,39}$ Elevated CSF protein was usually caused by the damage of bloodCSF barrier and increased protein synthesis in brain. Many diseases that obstructed the CSF pathway might elevate protein level, so the specificity of elevated protein level in CSF in late neurosyphilis was below $50 \%{ }^{13}$ There were $20.2 \%$ GPI patients with normal result of WBC and protein in CSF. Thus, the changes of WBC number and protein level were not specific for the neurosyphilis diagnosis. Accordingly, neurosyphilis, especially the late neurosyphilis, could not be excluded when the CSF routine test was normal.

Brain MRI is essential in the differential diagnosis of neurosyphilis. In this study, most GPI patients had brain atrophy, and many cases showed the atrophy in the temporal lobe, which was in accordance with a previous study. ${ }^{40}$ In view of the role of frontal and temporal lobes on cognition, a close correlation between brain atrophy and cognitive impairment was reported. ${ }^{30}$ It was verified that neuropil damage and neuronal loss were the main pathologic changes of cortical atrophy in GPI patients. ${ }^{41}$ In this study, only five cases had atrophy in the parietal lobe and no case showed atrophy in the occipital lobe. We should make a further study on the gray matter volume depending on voxel-based morphological (VBM) measurement. In this study, abnormal signal in MRI was common, which could help explain the various neurological symptoms and signs in GPI patients. It was reported that extensive T2 hyperintensity of white matter in the brain partly disappeared after penicillin treatment in a GPI patient. ${ }^{42}$ It was assumed that, in addition to microglia hypertrophy, gliosis, edema, cytotoxicity, and vascular occlusion might be involved in the formation of the abnormal signal, ${ }^{43}$ which might be resulted from NisslAlzheimer arteritis in the course of GPI. ${ }^{44}$ Thus, abnormal $\mathrm{T} 2$ hyperintensity could locate in the different brain regions. A further study on the cerebral perfusion for the GPI patients is much needed. In this study, 13 cases showed hydrocephalus, which was considered as an outcome of the block of
CSF evacuation due to the leptomeningeal thickening. Thus, for patients suffering from hydrocephalus who had cognitive impairment, leptomeningeal neurosyphilis should be taken into consideration.

\section{Limitations}

This study had the following limitations. It was a retrospective study, not all clinicians were aware of the symptoms and signs of GPI and some clinical data was missing. It might be overlooked. Furthermore, some scales such as MoCA and NPI could not replace a comprehensive assessment. And we did not use volumetric study such as medial temporal lobe atrophy (MTA) scale and VBM to provide quantitative analyses. It was not quite accurate in locating the atrophy in a certain lobe.

\section{Conclusion}

Syphilis is still prevalent today, we recommend effective and comprehensive sex education in the society. Increasing syphilis can increase the number of neurosyphilis cases. However, the symptoms and signs of GPI are complex, and easily misdiagnosed, the clinicians are still short of vigilance on neurosyphilis and should combine clinical manifestations, fluid changes and neuroimaging alteration to make a correct diagnosis of GPI. Early diagnosis is important in the prognosis of GPI due to the reversible dementia, so we suggest strengthening the syphilis curricula in the training program of the clinicians, and expanding syphilis screening, not only in the inpatient, but also in the outpatient, especially in the patient with cognitive impairment and neuropsychiatric symptoms.

\section{Acknowledgments}

The work was supported by the Research Fund of Beijing Ditan Hospital, Capital Medical University (DTQH201804); The National Key Research and Development Program of China (2016YFC1306300, 2016YFC1306000); The National Key R\&D Program of China-European Commission Horizon 2020 (2017YFE0118800-779238); The National Natural Science Foundation of China (81970992, 81571229, 81071015, 30770745); The Key Technology R\&D Program of Beijing Municipal Education Commission (kz201610025030); The Key Project of Natural Science Foundation of Beijing, China (4161004); The Natural Science Foundation of Beijing, China (7082032); Project of Scientific and Technological Development of Traditional Chinese Medicine in Beijing (JJ2018-48); Capital Clinical Characteristic Application Research (Z121107001012161). 


\section{Author Contributions}

All authors made a significant contribution to the work reported, whether that is in the conception, study design, execution, acquisition of data, analysis and interpretation, or in all these areas; took part in drafting, revising or critically reviewing the article; gave final approval of the version to be published; have agreed on the journal to which the article has been submitted; and agree to be accountable for all aspects of the work.

\section{Disclosure}

The authors report no conflicts of interest in this work.

\section{References}

1. Conde-Sendín MA, Amela-Peris R, Aladro-Benito Y, Maroto AA. Current clinical spectrum of neurosyphilis in immunocompetent patients. Eur Neurol. 2004;52(1):29-35. doi:10.1159/000079391

2. Daey Ouwens IM, Koedijk FD, Fiolet AT, et al. Neurosyphilis in the mixed urban-rural community of the Netherlands. Acta Neuropsychiatr. 2014;26(3):186-192. doi:10.1017/neu.2013.53

3. Zhang HL, Lin LR, Liu GL, et al. Clinical spectrum of neurosyphilis among HIV-negative patients in the modern era. Dermatology. 2013;226(2):148-156. doi:10.1159/000347109

4. Drago F, Merlo G, Ciccarese G, et al. Changes in neurosyphilis presentation: a survey on 286 patients. J Eur Acad Dermatol Venereol. 2016;30(11):1886-1900. doi:10.1111/jdv.13753

5. Li W, Jiang M, Xu D, et al. Clinical and laboratory characteristics of symptomatic and asymptomatic neurosyphilis in HIV-negative patients: a retrospective study of 264 cases. Biomed Res Int 2019;2019:2426313.

6. Zheng D, Zhou D, Zhao Z, et al. The clinical presentation and imaging manifestation of psychosis and dementia in general paresis: a retrospective study of 116 cases. J Neuropsychiatry Clin Neurosci. 2011;23(3):300-307. doi:10.1176/jnp.23.3.jnp300

7. Chen YY, Zhang YF, Qiu XH, et al. Clinical and laboratory characteristics in patients suffering from general paresis in the modern era. $J$ Neurol Sci. 2015;350(1-2):79-83. doi:10.1016/j.jns.2015.02.021

8. Janier M, Hegyi V, Dupin N, et al. 2014 European guideline on the management of syphilis. J Eur Acad Dermatol Venereol. 2014;28 (12):1581-1593. doi:10.1111/jdv.12734

9. Workowski KA, Bolan GA. Centers for disease control and prevention. Sexually transmitted diseases treatment guidelines, 2015. MMWR Recomm Rep. 2015;64(RR-03):1-137.

10. Creavin ST, Wisniewski S, Noel-Storr AH, et al. Mini-Mental State Examination (MMSE) for the detection of dementia in clinically unevaluated people aged 65 and over in community and primary care populations. Cochrane Database Syst Rev. 2016;(1):CD011145.

11. Zhang ZX, Zahner GE, Román GC, et al. Dementia subtypes in China: prevalence in Beijing, Xian, Shanghai, and Chengdu. Arch Neurol. 2005;62(3):447-453. doi:10.1001/archneur.62.3.447

12. Nasreddine ZS, Phillips NA, Bédirian V, et al. The Montreal Cognitive Assessment, MoCA: a brief screening tool for mild cognitive impairment. $J$ Am Geriatr Soc. 2005;53(4):695-699. doi:10.1111/j.1532-5415.2005.53221.x

13. Wormser GP, Pavia CS. Neurosyphilis. N Engl J Med. 2019;381 (24):2376-2377.

14. Daey Ouwens IM, Lens CE, Fiolet AT, Ott A, Koehler PJ, Verhoeven WM. Clinical presentation of general paralysis of the insane in a Dutch psychiatric hospital, 1924-1954. Eur Neurol. 2015;74(1-2):54-59. doi:10.1159/000435835
15. Tong ML, Chen YY, Zhu XZ, et al. Comparison of clinical and laboratory characteristics of general paresis and non-neurosyphilis dementia. Eur Neurol. 2018;80(1-2):82-86. doi:10.1159/000493866

16. Wang J, Guo Q, Zhou P, Zhang J, Zhao Q, Hong Z. Cognitive impairment in mild general paresis of the insane: AD-like pattern. Dement Geriatr Cogn Disord. 2011;31(4):284-290. doi:10.1159/ 000326908

17. Arando M, Caballero E, Curran A, Armengol P, Barberá MJ, VallMayans M. The epidemiological and clinical characteristics of the epidemic of syphilis in Barcelona. Actas Dermosifiliogr. 2019;110 (10):841-849. doi:10.1016/j.ad.2019.03.014

18. Carstairs C, Philpott B, Wilmshurst S. Sex education and the need for change. CMAJ. 2018;190(50):E1482-E1483. doi:10.1503/ cmaj. 180773

19. Wang X, Yang Y, Wang X, Li C. MRI findings and early diagnosis of general paresis of the insane. Neurol Res. 2014;36(2):137-142. doi:10.1179/1743132813Y.0000000273

20. Yanhua W, Haishan S, Le H, et al. Clinical and neuropsychological characteristics of general paresis misdiagnosed as primary psychiatric disease. BMC Psychiatry. 2016;16:230. doi:10.1186/s12888-0160925-3

21. Luo W, Ouyang Z, Xu H, Chen J, Ding M, Zhang B. The clinical analysis of general paresis with 5 cases. $J$ Neuropsychiatry Clin Neurosci. 2008;20(4):490-493. doi:10.1176/jnp.2008.20.4.490

22. Hagiya H, Deguchi K, Kawada K, Otsuka F. Neurosyphilis is a long-forgotten disease but still a possible etiology for dementia. Intern Med. 2015;54(21):2769-2773. doi:10.2169/internal medicine. 54.4956

23. Trzepacz PT, Hochstetler H, Wang S, Walker B, Saykin AJ. Alzheimer's disease neuroimaging initiative. relationship between the Montreal cognitive assessment and mini-mental state examination for assessment of mild cognitive impairment in older adults. $B M C$ Geriatr. 2015;15:107. doi:10.1186/s12877-015-0103-3

24. Glass L, Krueger F, Solomon J, Raymont V, Grafman J. Mental paper folding performance following penetrating traumatic brain injury in combat veterans: a lesion mapping study. Cereb Cortex. 2013;23 (7):1663-1672. doi:10.1093/cercor/bhs153

25. Friedrich F, Aigner M, Fearns N, Friedrich ME, Frey R, Geusau A. Psychosis in neurosyphilis - clinical aspects and implications. Psychopathology. 2014;47(1):3-9. doi:10.1159/000350059

26. Zhong X, Shi H, Hou L, et al. Neuropsychiatric features of neurosyphilis: frequency, relationship with the severity of cognitive impairment and comparison with alzheimer disease. Dement Geriatr Cogn Disord. 2017;43(5-6):308-319. doi:10.1159/000476060

27. Roberts MC, Emsley RA. Psychiatric manifestations of neurosyphilis. S Afr Med J. 1992;82(5):335-337.

28. Stober G, Ben-Shachar D, Cardon M, et al. Schizophrenia: from the brain to peripheral markers. A consensus paper of the WFSBP task force on biological markers. World J Biol Psychiatry. 2009;10 (2):127-155. doi:10.1080/15622970902898980

29. Egerton A, Fusar-Poli P, Stone JM. Glutamate and psychosis risk. Curr Pharm Des. 2012;18(4):466-478. doi:10.2174/ 138161212799316244

30. Russouw HG, Roberts MC, Emsley RA, Truter R. Psychiatric manifestations and magnetic resonance imaging in HIV-negative neurosyphilis. Biol Psychiatry. 1997;41(4):467-473. doi:10.1016/ S0006-3223(96)00060-1

31. Osman C, Clark TW. Tabes Dorsalis and Argyll Robertson Pupils. N Engl J Med. 2016;375(20):e40.

32. Shah BB, Lang AE. Acquired neurosyphilis presenting as movement disorders. Mov Disord. 2012;27(6):690-695. doi:10.1002/ mds. 24950

33. Murialdo A, Marchese R, Abbruzzese G, Tabaton M, Michelozzi G, Schiavoni S. Neurosyphilis presenting as progressive supranuclear palsy. Mov Disord. 2000;15(4):730-731. doi:10.1002/1531-8257(200007)15:4<730::AID-MDS1020>3.0.CO;2-O 
34. Martinelli P, Rizzo G, Scaglione C, Capellari S. Neurosyphilis orofacial dyskinesia: the candy sign. Mov Disord. 2013;28(2):246-247. doi: $10.1002 / \mathrm{mds} .25281$

35. Tang W, Huang S, Chen L, et al. Late neurosyphilis and tertiary syphilis in Guangdong Province, China: results from a cross-sectional study. Sci Rep. 2017;7:45339. doi:10.1038/srep45339

36. Marra CM, Maxwell CL, Smith SL, et al. Cerebrospinal fluid abnormalities in patients with syphilis: association with clinical and laboratory features. $J$ Infect Dis. 2004;189(3):369-376. doi:10.1086/ 381227

37. Jiang $\mathrm{Y}$, Weng R, Zhang $\mathrm{Y}$, et al. The performance of rapid plasma reagin (RPR) titer in HIV-negative general paresis after neurosyphilis therapy. BMC Infect Dis. 2018;18(1):144. doi:10.1186/s12879-0183062-4

38. Tuddenham S, Ghanem KG. Neurosyphilis: knowledge gaps and controversies. Sex Transm Dis. 2018;45(3):147-151. doi:10.1097/ OLQ.0000000000000723

39. Hooshmand H, Escobar MR, Kopf SW. Neurosyphilis. A study of 241 patients. JAMA. 1972;219(6):726-729.
40. Cai SN, Long J, Chen C, Wan G, Lun WH. Incidence of asymptomatic neurosyphilis in serofast Chinese syphilis patients. Sci Rep. 2017;7(1):15456. doi:10.1038/s41598-017-15641-w

41. Mao C, Gao J, Jin L, Peng B, Guo Y. Postmortem histopathologic analysis of neurosyphilis: a report of 3 cases with clinicopathologic correlations. J Neuropathol Exp Neurol. 2018;77:296-301. doi:10.1093/jnen/nly004

42. Alam F, Yasutomi H, Fukuda H, et al. Diffuse cerebral white matter T2-weighted hyperintensity: a new finding of general paresis. Acta Radiol. 2006;47(6):609-611. doi:10.1080/02841850600690389

43. Chen B, Shi H, Hou L, et al. Medial temporal lobe atrophy as a predictor of poor cognitive outcomes in general paresis. Early Interv Psychiatry. 2019;13(1):30-38.

44. Nagappa M, Sinha S, Taly AB, et al. Neurosyphilis: MRI features and their phenotypic correlation in a cohort of 35 patients from a tertiary care university hospital. Neuroradiology. 2013;55 (4):379-388. doi:10.1007/s00234-012-1017-9

\section{Publish your work in this journal}

Neuropsychiatric Disease and Treatment is an international, peerreviewed journal of clinical therapeutics and pharmacology focusing on concise rapid reporting of clinical or pre-clinical studies on a range of neuropsychiatric and neurological disorders. This journal is indexed on PubMed Central, the 'PsycINFO' database and CAS, and is the official journal of The International Neuropsychiatric Association (INA). The manuscript management system is completely online and includes a very quick and fair peer-review system, which is all easy to use. Visit http://www.dovepress.com/testimonials.php to read real quotes from published authors. 